\title{
Possible Means of Electrostatic Propulsion According to the Mbelek-Lachièze-Rey Scalar-Tensor Theory of Gravitation
}

\author{
F. O. Minotti ${ }^{1,2^{*}}$ \\ ${ }^{1}$ Universidad de Buenos Aires, Facultad de Ciencias Exactas Naturales, \\ Departamento de Física, Buenos Aires, Argentina \\ ${ }^{2}$ CONICET-Universidad de Buenos Aires, Instituto de Física del Plasma (INFIP), \\ Buenos Aires, Argentina \\ Received November 14, 2017; in final form, February 7, 2018
}

\begin{abstract}
As was shown recently, the scalar-tensor theory of gravitation proposed by Mbelek and Lachièze-Rey allows for a possible explanation of the forces reported in asymmetric microwave cavities. We show here that the theory in its revised version predicts a much simpler way of producing thrust by electrostatic means. We here briefly present the equations and derivations indicating that a constant force is predicted for a spherical capacitor with an asymmetric mass distribution, kept at constant voltage. Apart form other practical implications, this particular prediction, and a complementary proposal in which the spherical capacitor takes the place of the large mass in a Cavendish-like experiment, provides an additional possibility of experimentally testing this particular scalar-tensor gravitational theory.
\end{abstract}

DOI: $10.1134 / \mathrm{S} 0202289318030106$

\section{INTRODUCTION}

As has been reported, the scalar-tensor (ST) gravitational theory of Mbelek and Lachièze-Rey (MLR) [1], purports that electromagnetic (EM) fields do modify the space-time metric far more strongly than is predicted by general relativity or other ST theories. In the form presented in $[2,3]$ it was shown that the MLR theory could explain the forces on asymmetric resonant cavities reported in [4]. The mentioned experiments were intended to test the claim of propulsive, reactionless forces appearing in tuned asymmetric microwave cavities, rather than to test any particular theory. On the other hand, in [5] an experiment was performed with the aim of testing a particular prediction of the MRL theory, generation of pulsed gravitational forces by a transient magnetic field, with very clear, positive results. In fact, the effect measured in [5] could be in principle engineered as an alternative way of propulsion. In the present communication we show that the revised version of the theory [3] allows for still another alternative propulsion system, employing only electrostatic means. Moreover, the characteristics of the proposed device makes it apt as an additional test of the MLR theory.

\footnotetext{
${ }^{*}$ E-mail: minotti@df .uba.ar
}

Related to this point, there is the question of the constant $\Gamma$ of the theory, introduced below. It is important to mention that the value of $\Gamma$ used in [3] is a factor about $10^{-4}$ of that derived in [1] and used in [2], and was determined by fitting the measured discordant values of Newton's gravitational constant $G$ in different laboratories, with those predicted from a particular nonlinear solution for the internal scalar $\phi$ of the theory, generated by the magnetic field of the Earth. The same procedure was employed originally in [1], but with a different solution, corresponding to the linearized equations, to obtain the value of $\Gamma$ used in [2]. The solution for $\phi$ used in [1] presents a better chi-squared value for the fit than that used in [3], whereas the solution used in the latter work is introduced as a possible resolution to the problem of much too strong gravitational effects by the Earth's magnetic field as predicted by the linearized version of theory.

On the other hand, the original value of $\Gamma$ used in [2] gave a good reproduction of the results of forces on microwave cavities reported at that time by the group at the Northwestern Polytechnical University of China [6]. However, in later, more refined experiments by the same group [7], a much smaller upper limit on the forces was reported, consistent with the smaller value of $\Gamma$ in [3], the value which is also 
consistent with the actual forces on resonant cavities measured by the group at NASA [4].

It is thus of interest that the proposal in this work provides a possible alternative experimental determination of the constant $\Gamma$ of the theory.

Finally, it is also shown that, as required for reactionless propulsion, the weak energy condition is violated in this case.

\section{BASIC EQUATIONS}

The details of the considered MLR ST theory were already presented in $[2,3]$, so only the necessary equations corresponding to the weak-field (WF) approximation are recalled.

In the WF approximation the geodesic equation for neutral matter is equivalent to an equation of motion due to the action of a 3D specific force $\mathbf{f}$ (per unit mass) in flat Minkowski space-time, derived form a potential $\chi$ :

$$
\mathbf{f}=-\nabla \chi
$$

whose determining equation with EM sources is $\left(\square \equiv c^{-2} \partial^{2} / \partial t^{2}-\nabla^{2}\right)$

$$
\square \chi=\frac{\partial^{2} \phi}{\partial t^{2}}+c^{2} \Gamma\left(B^{2}-E^{2} / c^{2}\right),
$$

where $E$ and $B$ are the magnitudes of the electric and magnetic fields, respectively, and $\Gamma=3.35 \times$ $10^{-12} \mathrm{~A}^{2} \mathrm{~N}^{-2}[3]$.

For a pure electrostatic field the equation for $\chi$ thus reduces to

$$
\nabla^{2} \chi=\Gamma E^{2} .
$$

Due to the bearing that they have in the present situation, we recall at this point the arguments used in [3] for the case of a magnetostatic field. As can be seen in the second equation after Eq. (13) in [2], for static cases the scalar sources of the force $\mathbf{f}$ are proportional to the Laplacians of $\phi$ and of $\psi$. As shown further in [3], $\nabla^{2} \psi$ is proportional to $\nabla^{2} \phi$, so that regions with $\nabla^{2} \phi=0$ do not contribute to the force. This is crucial in order for the MLR theory to be consistent with the lack of strong gravitational effects due to the magnetic field of the Earth. In a magnetostatic case the nonlinear equation for $\phi$ is

$$
\nabla^{2} \phi+\nabla \phi \cdot \nabla \phi=2 \Gamma B^{2},
$$

which, outside the distributions of electric currents, admits the solution $\nabla \phi=\sqrt{2 \Gamma} \mathbf{B}$, so that $\nabla^{2} \phi=0$. However, for an electrostatic field, the corresponding equation is

$$
\nabla^{2} \phi+\nabla \phi \cdot \nabla \phi=-2 \Gamma E^{2} / c^{2} .
$$

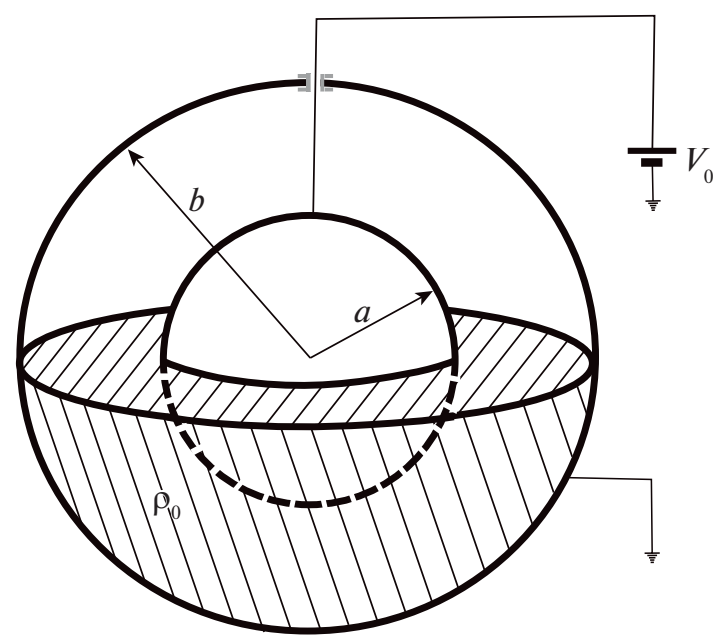

Fig. 1. Sketch of the spherical capacitor with its internal asymmetric distribution of a dielectric of mass density $\varrho_{0}$, the internal conductor of radius $a$ at potential $V_{0}$, and a grounded external conductor of radius $b$.

The minus sign in the right-hand side of this equation precludes the existence of solutions with $\nabla \phi$ proportional to the electrostatic field $\mathbf{E}$ outside charge distributions, so that the equation at the leading order is

$$
\nabla^{2} \phi=-2 \Gamma E^{2} / c^{2},
$$

thus resulting in (3).

A further important point is that in order to achieve reactionless thrust, effective negative energy densities are required $[8,9]$. This represents a violation of the weak energy condition (WEC). The WEC can be cast in the case considered as $\nabla^{2} \phi \geqslant 0$ (see Eq. (33) in [2]), which is clearly violated by Eq. (4).

\section{FORCE ON A SPHERICAL CAPACITOR WITH AN ASYMMETRIC MASS DISTRIBUTION}

We now determine the force acting on a spherical capacitor, sketched in Fig. 1. Taken the potential at the inner conductor of radius $a$ to be $V_{0}$, and the outer conductor of radius $b$ to be at zero potential, the electrostatic field for $a<r<b$ is given by (there is only a radial component)

$$
E=\frac{V_{0} a b}{(b-a) r^{2}}
$$

which, when employed in Eq. (3), with the condition at $r=a$ that $f_{r}=-d \chi / d r=0$ (due to continuity of the force, that is zero for $r<a$ ), results in a radial specific force for $a<r<b$ given by

$$
f_{r}=-\frac{\Gamma V_{0}^{2} a b^{2}}{(b-a)^{2} r^{2}}\left(1-\frac{a}{r}\right),
$$


while for $r>b$ it is

$$
f_{r}=-\frac{\Gamma V_{0}^{2} a b^{2}}{(b-a)^{2} r^{2}}\left(1-\frac{a}{b}\right) .
$$

The total force on the capacitor is thus given by the integral

$$
\mathbf{F}=\int \rho \mathbf{f} d V
$$

extended to the whole volume occupied by the mass density distribution $\rho$. Of course, if $\rho$ has spherical symmetry, the resulting force is zero. We thus consider that the lowest half contains a dielectric of mass density $\varrho_{0}$ in $a<r<b$, and vacuum or a very light dielectric medium in the other half (it is easily shown that this distribution maximizes the total force), while the rest of the mass distribution, either internal or external, is spherically symmetric. The resulting force is then directed along the axis of symmetry of the mass distribution (which we designate as $z$, with $z$ growing upward):

$$
F_{z}=-\frac{\pi \Gamma \rho_{0} V_{0}^{2} b^{2}}{(b / a-1)^{2}}\left[1+\ln \left(\frac{b}{a}\right)-\frac{b}{a}\right] .
$$

As can be seen from (7), the larger $\rho_{0} V_{0}^{2} b^{2}$ and the smaller $b / a$, the better it is for force maximization.

As an example of the expected values of thrust let us consider a spherical capacitor with $a=4 \mathrm{~cm}$, $b=5 \mathrm{~cm}, \rho_{0}=2 \mathrm{~g} \mathrm{~cm}^{-3}$, and $V_{0}=5 \mathrm{kV}$. The force predicted by Eq. (7) is $F_{z}=565 \mu \mathrm{N}$. Considering that in this example we have employed rather conservative parameters, an experimental verification of the predicted effect could be accomplished with relative ease. On the other hand, if the original value of $\Gamma$ were employed, the force would be of about $5 \mathrm{~N}$, approximately twice the weight of the massive dielectric filling half the space between the spheres. It is hard to imagine that such a strong effect appearing in asymmetric capacitors or on objects nearby high voltage capacitors could go unobserved. Assuming the validity of the theory, it is thus expected that either the smaller value of $\Gamma$ is closer to the correct one, or that a mechanism analogous to that argued in [3] to exist in the case of a magnetostatic field, acts to prevent strong effects in an electrostatic case as well. The latter alternative would require a full revision of the arguments presented in [3]. It is thus very important to be able to perform the proposed experiment.

\section{EXPERIMENT ANALYSIS}

A sketch of the proposed experiment can be seen in Fig. 2a). Since the external conductor is closed and grounded, and the charging of the internal conductor does not change the total charge of the system,
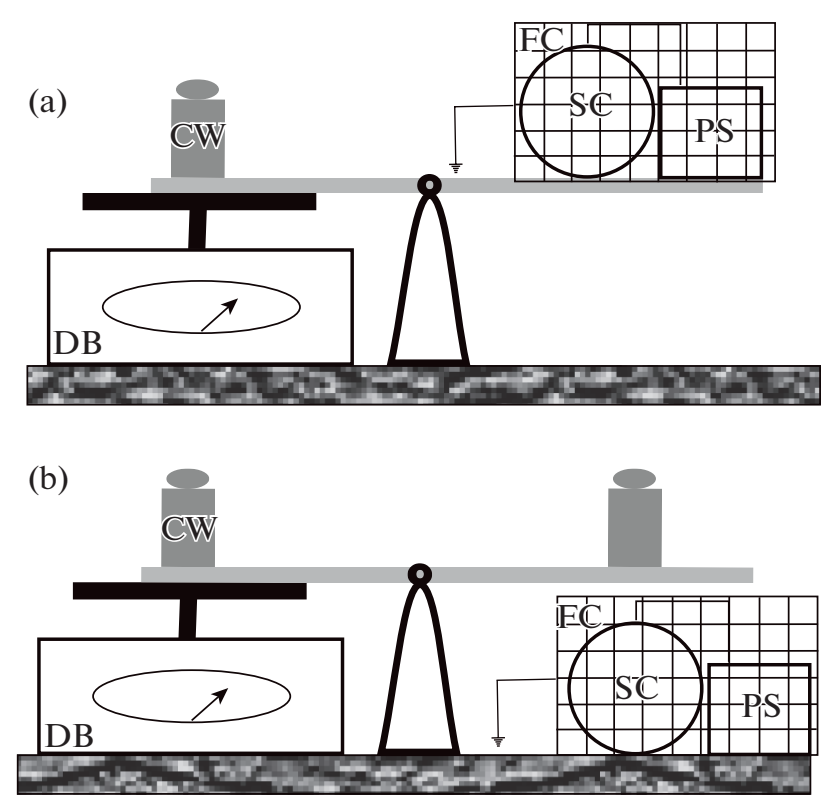

Fig. 2. (a) Sketch of the experimental arrangement for thrust measurement showing the spherical capacitor (SC), power source (PS), digital balance (DB), counterweight (CW) and Faraday cage (FC). (b) Sketch of the experimental arrangement for a Cavendish-like measurement, where the test mass is designated (TM).

forces of electric origin between the capacitor and any external medium, including the surrounding air, do not change when the capacitor is polarized, and so do not interfere with the actual measurement. Spurious forces can appear, however, if a near conductor changes its potential during the measurement, as is a case of the external section of the wire polarizing the internal conductor. To avoid this problem, one can integrate a light-weight, battery cell powered source in a single item with the capacitor as the load of the measuring balance, since only a low-power source is required. This item can be further included in a Faraday cage for better isolation from spurious electric forces. Turning on and off of the power source can be accomplished with a preset electronic timer integrated with the source itself. Under these conditions a forseeable source of spurious effects is a possible variation of the center of mass of the system due to internal electric stresses. Minimization of this effect requires a careful tightening of all components. Moreover, identification and evaluation of the magnitude of this effect can be achieved by repeating the measurement with the item rotated at different angles about a vertical axis.

A complementary measurement can also be obtained using the result of the theory that the spherical capacitor also generates an external specific force given by Eq. (6), using, for example, the arrangement sketched in Fig. 2b). Note that, from the dependence 
of the external force on the distance to the capacitor center, the gravitational effect of the capacitor is predicted to be completely equivalent to that of a spherical mass of the value

$$
M=\frac{\Gamma V_{0}^{2} a b^{2}}{(b-a)^{2} G}\left(1-\frac{a}{b}\right),
$$

where $G$ is Newton's gravitational constant.

For the parameters of the capacitor used above: $a=4 \mathrm{~cm}, b=5 \mathrm{~cm}$, and $V_{0}=5 \mathrm{kV}$, the equivalent mass is $M=2.5 \times 10^{6} \mathrm{~kg}$. This large value indicates that a Cavendish-like measurement should very easily discriminate the existence or not of the effect. Note that in this case, in which the spherical capacitor takes the place of the large mass in the Cavendish experiment, an asymmetric distribution of the internal dielectric is not required.

\section{CONCLUSIONS}

We have shown that the MLR theory predicts that a relatively simple device, a spherical capacitor at constant voltage with an asymmetric mass distribution, can generate measurable forces which can in principle be scaled up rather easily. The fact that the device is a closed capacitor with its external surface grounded eliminates many sources of spurious effects, such as ion wind, electric interactions with external objects, etc., thus making the experiments more easy an accurate. Complementarily, a Cavendish-like experiment, in which the large mass is replaced by a spherical capacitor, should clearly denote the existence or not of the effect. Given the theoretical and practical consequences implied, experimental tests of the theory here reported are highly desirable and strongly encouraged.

\section{REFERENCES}

1. J. P. Mbelek and M. Lachièze-Rey, Grav. Cosmol. 8, 331 (2002).

2. F. O. Minotti, Grav. Cosmol. 19, 201 (2013).

3. F. O. Minotti, Grav. Cosmol. 23, 287 (2017).

4. H. White, P. March, J. Lawrence, J. Vera, A. Sylvester, D. Brady, and P. Bailey, J. Prop. Power 33, 830 (2017).

5. J. P. Mbelek, Grav. Cosmol. 21, 340 (2015).

6. J. Yang, Y.-Q. Wang, Y.-J. Ma, P.-F. Li, L. Yang, Y. Wang, and G.-Q. He, Chin. Phys. B 22, 050301 (2013).

7. J. Yang, X.-C. Liu, Y.-Q. Wang, M.-J. Tang, L.-T. Luo, Y.-Z. Jin, and Z.-X. Ning, J. Prop. Tech. 37, 362 (2016).

8. C. Barcelo and M. Visser Class. Quantum Grav. 17, 3843 (2000).

9. F. S. N. Lobo and M. Visser, Class. Quantum Grav. 21, 5871 (2004). 